\title{
Beyond the Child-Langmuir law: A review of recent results on multidimensional space-charge-limited flow ${ }^{\text {a) }}$
}

\author{
J. W. Luginsland ${ }^{\text {b) }}$ \\ Center for Plasma Theory and Computation, High Power Microwave Division, \\ Directed Energy Directorate, Air Force Research Laboratory, Kirtland AFB, New Mexico 87117 \\ Y. Y. Lau \\ Department of Nuclear Engineering and Radiological Sciences, University of Michigan, Ann Arbor, \\ Michigan 48109-2104 \\ R. J. Umstattd ${ }^{\mathrm{c})}$ \\ Center for Plasma Theory and Computation, High Power Microwave Division, \\ Directed Energy Directorate, Air Force Research Laboratory, Kirtland AFB, New Mexico 87117
}

J. J. Watrous

NumerEx, Albuquerque, New Mexico 87106

(Received 31 October 2001; accepted 15 January 2002)

\begin{abstract}
Space-charge-limited (SCL) flows in diodes have been an area of active research since the pioneering work of Child and Langmuir in the early part of the last century. Indeed, the scaling of current density with the voltage to the 3/2's power is one of the best-known limits in the fields of non-neutral plasma physics, accelerator physics, sheath physics, vacuum electronics, and high power microwaves. In the past five years, there has been renewed interest in the physics and characteristics of SCL emission in physically realizable configurations. This research has focused on characterizing the current and current density enhancement possible from two- and three-dimensional geometries, such as field-emitting arrays. In 1996, computational efforts led to the development of a scaling law that described the increased current drawn due to two-dimensional effects. Recently, this scaling has been analytically derived from first principles. In parallel efforts, computational work has characterized the edge enhancement of the current density, leading to a better understanding of the physics of explosive emission cathodes. In this paper, the analytic and computational extensions to the one-dimensional Child-Langmuir law will be reviewed, the accuracy of SCL emission algorithms will be assessed, and the experimental implications of multidimensional SCL flows will be discussed. () 2002 American Institute of Physics.
\end{abstract}

[DOI: $10.1063 / 1.1459453$ ]

\section{INTRODUCTION}

Space-charge-limited (SCL) flows in diodes have been an area of active research since the pioneering work of Child and Langmuir ${ }^{1,2}$ in the early part of the last century. Indeed, the scaling of current density with the voltage to the 3/2's power is one of the best-known limits in the fields of nonneutral plasma physics, accelerator physics, sheath physics, vacuum electronics, and high power microwaves. Refinements to the basic scaling of current density to voltage and gap spacing, such as finite initial velocity, ${ }^{3}$ relativistic applied voltage, ${ }^{4}$ and quantum mechanical effects ${ }^{5}$ have further increased the scope of the theory. The physics of SCL flows and emission appear throughout the literature of plasma physics. These theories, however, focus typically on SCL flows in one-dimension only. This paper reviews recent attempts to extend the description to two dimensions. ${ }^{6-10} \mathrm{We}$ note that analytic descriptions of two-dimensional (2-D) beam transport are available ${ }^{11}$ as are investigations into the effect of 2-D geometrical cathode surface features, ${ }^{12}$ but the

\footnotetext{
${ }^{a)}$ Paper KI2 2, Bull. Am. Phys. Soc. 46, 175 (2001).

${ }^{b}$ Invited speaker. Present address: SAIC, Albuquerque, NM.

${ }^{c}$ Present address: The Naval Postgraduate School, Monterey, CA.
}

seemingly simple problem of 2-D planar SCL emission remains unsolved. We focus on classic SCL emission, where the normal component of the electric field is forced to zero at the cathode due to a large reservoir of free electrons at the emitter surface. While other models of emission for which this condition is not a requirement do exist, we do not consider them explicitly here. Furthermore, this paper concentrates only on electrostatic, single species behavior. We stress that the relatively simple formulation of electrostatic multidimensional SCL flows belies the rich physics present in this ubiquitous phenomena.

The work presented herein is motivated by two distinct, but related goals. First, these models are useful for accurately simulating a wide variety of high voltage devices. Emission algorithms are critical to this process, and a wide variety of models have been developed for this work ${ }^{13,14}$ and implemented in multidimensional codes. To assess how well such algorithms work, a theoretical prediction of SCL behavior is needed. ${ }^{14}$ The second goal focuses on the practical design of experimental cathodes in vacuum electronics, high power microwave devices, and spacecraft thrusters. The large currents (from tens of amperes to tens of kiloamperes) and current densities (up to $1 \mathrm{kA} / \mathrm{cm}^{2}$ ) place harsh requirements on 


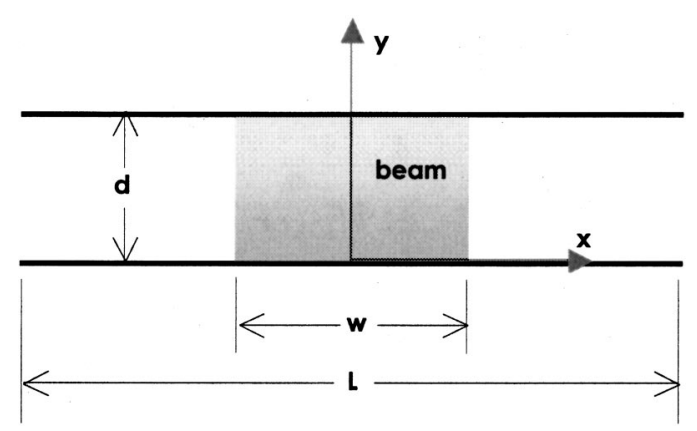

FIG. 1. Configuration of the finite-width beam in parallel-plate geometry. Note that the problems coordinate system corresponds to the boundary conditions listed in Eq. (4).

the cathode design and material present in high voltage devices. Advanced cathode designs are proving crucial to limiting damage and plasma formation ${ }^{15}$ as well as providing high brightness, uniform beams ${ }^{16}$ for the next generations of rf devices, accelerators, and spacecraft thrusters.

The classical, one-dimensional, nonrelativistic theory for SCL emission starts by combining energy conservation with both continuity and Poisson's equation in one dimension to give the following equation in MKS units:

$$
\frac{d^{2} \phi}{d y^{2}}=\frac{J}{\varepsilon_{0}} \frac{1}{\sqrt{\frac{2 e}{m} \phi(y)}}
$$

with boundary conditions that the voltage drop $V$ exists between the cathode $(y=0)$ and the anode $(y=d)$ and the electric field on the cathode $E(y=0)$ is zero. This last condition assumes that the SCL current density is reached when the self-electric fields of the electrons shield out the applied voltage. The quantities $\phi(y)$ and $J$ are the electrostatic potential and the current density, respectively. Furthermore, the quantities $-e$ and $m$ are the electron charge and mass while $\epsilon_{0}$ is the free space permitivity. Equation (1) yields the familiar Child-Langmuir result

$$
J=J_{\mathrm{CL}}=\frac{4}{9} \varepsilon_{0} \sqrt{\frac{2 e}{m}} \frac{V^{3 / 2}}{d^{2}}
$$

showing the strong bond between current density and the boundary data. Equation (1) has no solution for a current density greater than that specified by Eq. (2). Since the problem explicitly assumes no time dependence, the ChildLangmuir current can be regarded as the largest current that can be emitted without time-dependent behavior manifesting itself. Now consider the following two-dimensional problem where we have parallel plate geometry with gap separation $d$, but only a strip of width $w$ emits on a cathode of length $L$ (see Fig. 1). The description of this problem can be formulated in a similar manner by combining energy conservation, continuity, and the Poisson equation with an assumption that the electron motion is restricted in the $y$ direction by an infinite magnetic field. ${ }^{7}$ This last assumption simplifies the mathematics so that the following system of equations can be written down:

$$
\begin{aligned}
& \nabla^{2} \phi(x, y)=0 ; \quad|x|>w / 2, \\
& \nabla^{2} \phi(x, y)=\frac{1}{\varepsilon_{0}} \frac{J(x)}{\sqrt{\frac{2 e}{m} \phi(x, y)}}, \quad|x| \leqslant w / 2
\end{aligned}
$$

with boundary conditions that the voltage drop $V$ exists between cathode and anode and the cathode surface electric field due to the space charge at the limiting current is zero. Finally, we close Eq. (3) with the assumption that the electric field on the transverse boundaries is the vacuum field. Another option for these boundaries is a Neumann boundary condition, although this does not significantly modify the solution if the transverse boundaries are spatially removed from the beam region. These boundaries conditions are given mathematically by

$$
\begin{aligned}
& \phi(x, y)=0, \quad y=0, \quad|x|<L / 2, \\
& \phi(x, y)=V, \quad y=d, \quad|x|<L / 2, \\
& \frac{\partial \phi(x, y)}{\partial y}=0, \quad y=0 ; \quad|x|<w / 2, \\
& \phi(x, y)=V\left(\frac{y}{d}\right), \quad|x|=L / 2 .
\end{aligned}
$$

So, the two-dimensional problem under consideration is an electrostatic, elliptical, steady state problem with parallel plate geometry and motion restricted to one dimension by a strong magnetic field. This isolates the fundamental, single species, SCL emission value without geometrical, ${ }^{12}$ electromagnetic, ${ }^{17}$ or transient effects. ${ }^{10}$

The rest of the paper is organized as follows. Section II looks at the solution of Eqs. (3) and (4) under the assumption of spatially constant current density. Section III relaxes this assumption, and thereby describes the mechanism for producing large current density enhancements near the edge of the emitter. Section IV assesses the experimental impact of these high current density regions, especially on the standard description of explosive emission, and Sec. V offers conclusions and avenues for future research.

\section{SPATIALLY CONSTANT CURRENT DENSITY STRIP}

We start our study of Eqs. (3) and (4) by assuming that the current density in Eq. (3) is constant [i.e., $J(x)=J_{0}$ ]. Recent particle-in-cell (PIC) calculations ${ }^{6}$ have shown that the true limiting current for this case can exceed the onedimensional limiting current by a significant degree. These calculations used an emission method called "overinjection." A current density, that is constant over the entire strip $w$, is emitted into the diode. This current density $J$ is progressively increased until a virtual cathode is observed. In other words, the calculations are repeated until the greatest current is found that allows the beam to propagate without reflected particles. This current is then interpreted as the limiting current as it satisfies the original expectations of Child and Langmuir, namely the largest current with laminar, steady-state flow. We should stress that this case does not solve the full set of 2-D boundary conditions as no attempt is 


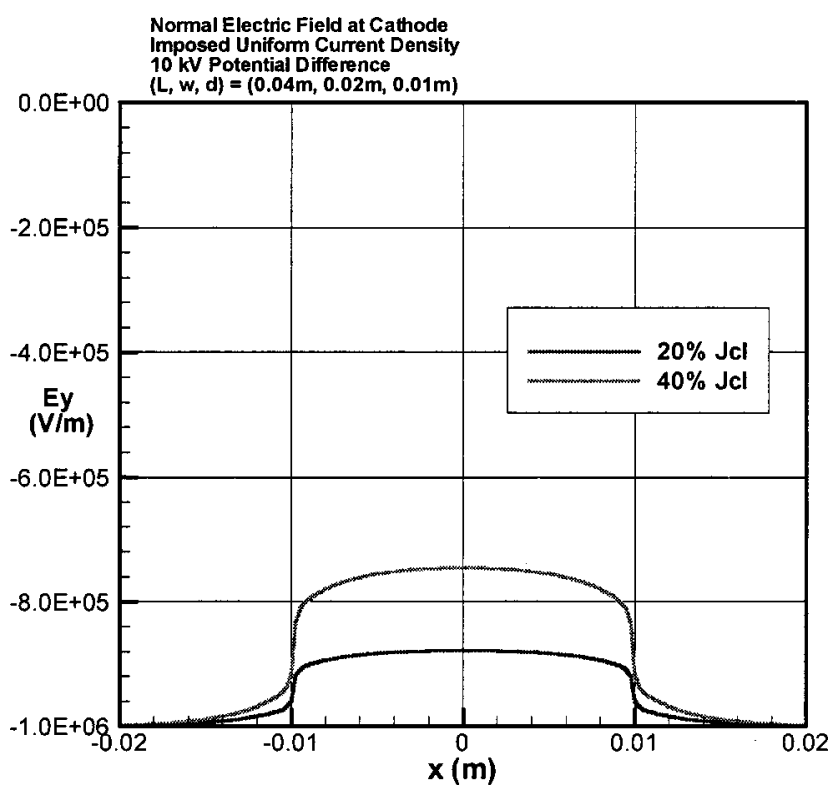

FIG. 2. Cathode electric field for different uniform current density profiles.

made to satisfy Eq. (4c) everywhere along the emitting strip. However, this case does have relevance to various physical emission mechanisms, such as thermionic and photoemission, where the maximum emitted current density is specified by conditions other than the applied voltage (i.e., when not running completely space charge limited).

The enhancement in constant current density that can be injected over a finite strip on the cathode without virtual cathode formation can be synthesized from the PIC data in the following empirical scaling law: ${ }^{6}$

$$
\frac{J_{\mathrm{CL}}(2-\mathrm{D})}{J_{\mathrm{CL}}(1-\mathrm{D})}=1+\frac{0.3145}{w / d}+\frac{0.0004}{(w / d)^{2}}
$$

for $w / d$ as small as 0.1. Equation (5) clearly recovers the Child-Langmuir result in the one-dimensional limit as $w / d$ goes to infinity. Lau recently derived a similar result from first principles for the case of $w / d$ on the order of one or greater where the last term in Eq. (5) is negligible. ${ }^{9}$ For a planar strip, he found $J_{\mathrm{CL}}(2-\mathrm{D}) / J_{\mathrm{CL}}(1-\mathrm{D})=1+(d / w \pi)$. The agreement with Eq. (5) is excellent. Furthermore, he extended the theory to describe other geometries, the most important being a circular patch of radius $R$. In this case, the scaling becomes $J_{\mathrm{CL}}(2-\mathrm{D}) / J_{\mathrm{CL}}(1-\mathrm{D})=1+(d / 4 R)$. PIC calculations similar to Ref. 6 verified this scaling for $R / d$ on the order of one and greater. Equation (5) and the analytic results presented herein given valuable rules of thumb for the onset of virtual cathode formation in two-dimensional flows.

\section{SPATIALLY NONUNIFORM CURRENT DENSITY STRIP}

The issue with these approaches is that they do not allow the current density to be nonuniform. As noted previously, this has the consequence that, unlike the one-dimensional equivalent, the constant current density problem does not require the cathode electric field to be zero everywhere on the emitting surface. ${ }^{7}$ This is clearly at odds with our intu- ition for space-charge-limited flow. Consider the electric field on the cathode when solving the constant current density case as shown in Fig. 2. The electric field is reduced at the center of the flow in keeping with the space-charge effect being maximized at this location. We note that when virtual cathodes form for this case, the initial reflection also occurs at the center of the beam while the edge flow continues to the anode without reflection. ${ }^{6}$ Experimental evidence of this is also seen in photoemission experiments when the laser intensity is sufficient to provide over-injection at the center of the beam while the edges of the beam continue to flow unreflected. $^{10}$

These issues motivate the solution to Eqs. (3) and (4) with the full spatial nonuniformity in the emitted current density $J(x)$ retained. ${ }^{7}$ Equations (3) and (4), however, have a number of mathematical and numerical difficulties. The most obvious impediment to analytic progress is the nonlinearity in Eq. (3). A less obvious difficulty is the current density is not specified. As in the one-dimensional case solved by Child and Langmuir, the current density must be strongly associated with the boundary data on the cathode in the beam region. While an analytic solution for a spatially nonuniform current density strip remains to be found, a numerical technique has been developed that simultaneously determines the current density and the electrostatic potential consistent with the boundary conditions. This numerical method is described here.

The solution technique is made of two separate iteration loops. ${ }^{7}$ In the first part, we solve for the electrostatic potential consistent with a specified current density $J(x)$. In this iteration, we only apply the Dirichlet boundary condition (i.e., the voltage) on the cathode in the beam region. We use a standard numerical technique by discretizing the computational domain with a rectangular grid. The grid spacing need not be the same in the longitudinal direction as the transverse. Poisson's equation is then solved iteratively in finite difference form on a typical five-point stencil.

The second part of the technique occurs after the solution to the electrostatic potential is obtained. In this loop, we numerically implement the second cathode boundary condition where the normal electric field in the beam region is forced to zero. We force the Neumann condition by solving iteratively for a current density $J(x)$ that drives the surface field to zero. We do this on a cell-by-cell basis where we use the cathode surface electric field information to derive a current density that will drive the cell in question to zero. The algorithm for this is similar to using the one-dimensional solution to Eq. (1) to find the surface electric field for a given $J{ }^{18}$ and using a secant method to extrapolate to the current density that drives the field to zero.

Numerically, the procedure starts with a uniform current density with the Child-Langmuir value as an initial guess. We then solve the restricted Poisson problem where we obtain the potential for the given $J(x)$ where we only apply the Dirichlet boundary condition for the cathode in the beam region. This can be thought of as the inner iteration. We then perform an outer iteration to modify $J(x)$ to drive the surface electric field to zero in the cathode beam region. Hence, the Neumann boundary condition is satisfied in an evolutionary 

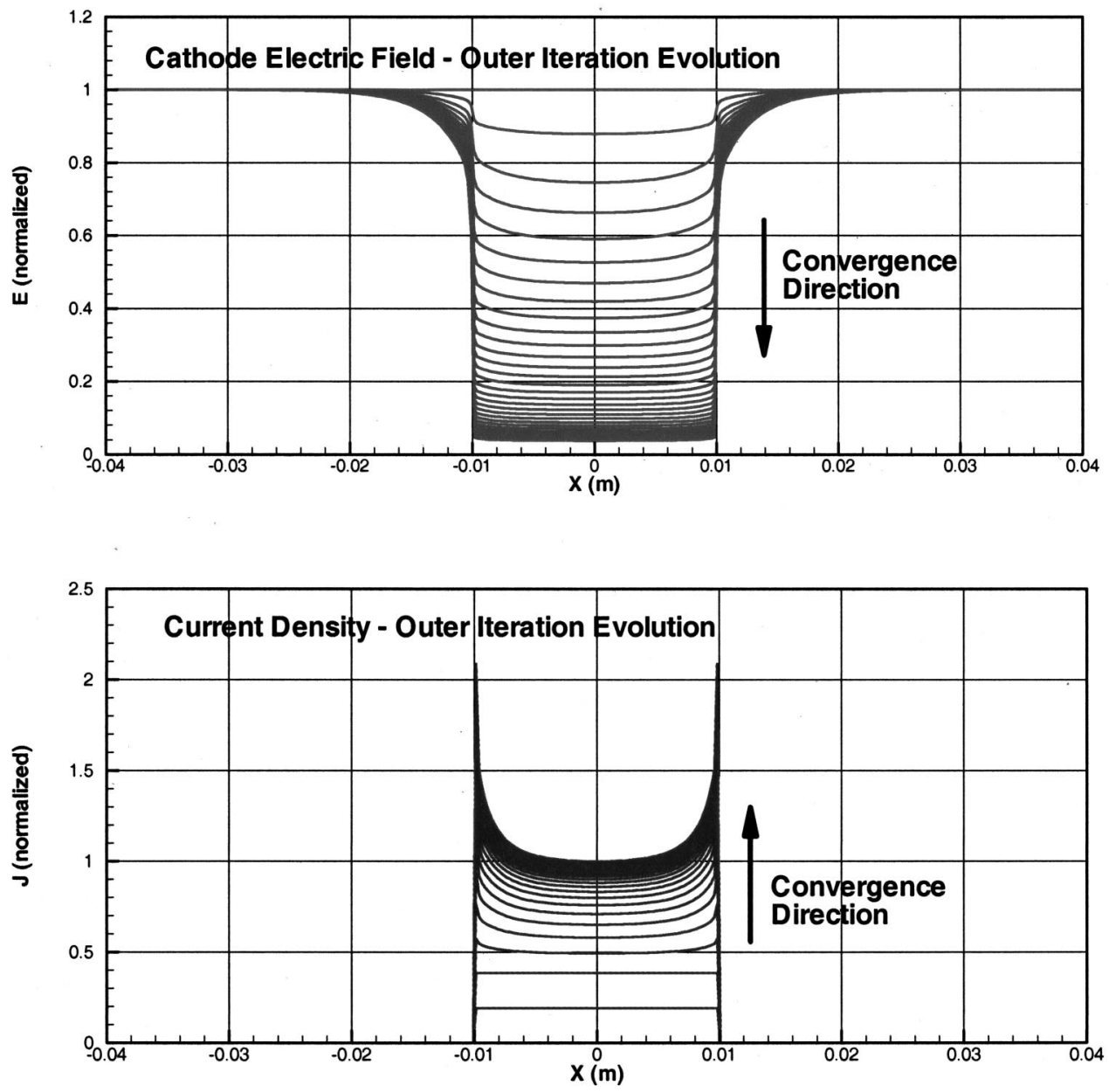

FIG. 3. Evolution of the cathode electric field and current density profile through iterative numerical solution to the 2-D SCL emission problem.

sense, where successive results from the inner calculation are used to build the current density profile that drives the normal electric field to zero due to the presence of the beam. The reader is referred to Ref. 7 for the full details of the method.

The evolution of the cathode electric field and the current density profile builds an interesting picture, shown in Fig. 3. The current density, assumed to be uniform initially, grows pronounced peaks or "wings" on either side while the electric field, which is initially nonuniform and nonzero, quickly evolves to a small value along the entire cathode surface. The convergence criterion used for the numerical scheme is that the surface electric field is less than $5 \%$ of the vacuum value $(V / d)$ everywhere in the cathode beam region. Stricter tolerances do not significantly change the current density amplitudes or profiles. ${ }^{7,18}$

\section{RESULTS}

In Fig. 3, the current density profile for the converged solution has a universal shape when we normalize the current density by the Child-Langmuir value and we normalize the position by the gap separation $d .^{7,8}$ Qualitatively similar wing structures have been observed experimentally in the Naval Research Laboratory's Electra diode. ${ }^{19}$ In general, when the beam width to gap ratio $w / d$ is on the order of one or greater, we see a wing structure on either end while the intervening regions have approximately the same value as the one-dimensional solution. When $w / d$ is less than one, however, the peak current density in the wing increases as does the value of the current density between the wings. ${ }^{7,8}$ We interpret this phenomenon as follows: the intense space charge in the wing structure compensates for the absence of charge beyond the emitter edge. In essence, the wing can be thought of as mimicking the space charge necessary to simulate the one-dimensional condition. When we go to small $w / d$, however, there is simply not enough space charge in the wing to force the surface electric field to zero. Thus, the current density over the entire strip must increase.

Despite this highly peaked structure in the current density, the total current for all these calculations compares well with the scaling presented in Eq. (5) for $w / d$ on the order of one. ${ }^{7}$ This is due to the fact that current carried in the wing structure is negligible compared to the current carried in the bulk of the beam. The 2-D peak current density, however, can exceed the Child-Langmuir current density by over a factor of 3.,8 This feature can cause considerable problems in experiments as the amount of damage increases due to the enhanced $J$. Furthermore, the peak current density shows greater variation and does not appear to be a simple function of $w$ and $d$. Indeed, the numerical methods (which show 
weakly divergent results for very small emitting strips), in addition to the analytic results presented earlier, all break down for small $w / d(\ll 1)$, and this remains an area of active research. ${ }^{9}$ It is critical to establish the asymptotic limits as $w$ goes to zero, especially when one considers the sharp fieldemitting tips and velvet tufts currently in use in many cathodes today.

We stress that unlike the planar structures that we have discussed to this point, multidimensional effects in arrays of small $w / d$ emitters, with highly peaked wing structures, can significantly affect the total current drawn. To illustrate how these increased current density wings might affect total current, we suppress emission from some portion of the cathode in two different fashions and monitor changes in the total current. ${ }^{8}$ In the first method, we divide a baseline four-cmwide emission region into 40 separate, equal-length subregions. As we systematically decrease the portion of each subregion that is allowed to emit, little effect is seen on the total current emitted until nearly all of the cathode emission area has been turned off. Almost $80 \%$ of the full emission area current can be supplied by a mere $20 \%$ of the emitting cathode area due to the ability of the enhanced current density wings to compensate for the paucity of emission area. ${ }^{8}$ In the second method, this effect was further examined by suppressing emission from only a central portion of the cathode. The nonemitting portion was gradually increased in size as the total current was monitored. The two sets of wings present at the edges of the nonemitting portion cannot compensate for the nonemitting area nearly as well as the 40 sets of wings available in the discrete patches case. Such an effect has direct relevance to understanding space-chargelimited emission from explosive emission cathodes, plasma cathodes, ferroelectric cathodes, photocathodes, and even thermionic cathodes (if operating well above the temperature-limited regime). Many small portions of the emission surface may be completely inactive before a significant change is detected in observed total current. If the emission is being provided by numerous microsites, many such sites could be turning off and on multiple times during the life of a given cathode with little or no effect on the observed total current. ${ }^{8}$

One goal of this research was the development of a test problem for emission algorithms implemented in other software. Because our numerical routine solves the 2-D SCL emission problem in a robust and well-understood manner, we used these solutions as benchmarks for evaluation of both particle-in-cell and gun code emission algorithms. The agreement with PIC time domain dual cell emission algorithms ${ }^{14}$ and steady state, ray tracing, finite element emission ${ }^{20}$ is excellent if the wing structure is sufficiently resolved by the grid in question. Therefore, we can relax our planar geometry assumption and study the effects of multidimensional emission in physically relevant structures using the 2-D code TRAK. ${ }^{8,20}$ One important point that comes out of this analysis is that small Pierce focusing electrodes, on the order of $1 / 100$ th of the gap spacing, can greatly reduce the wing structure by compensating for the "missing" space charge at the beam edge. This occurs by reducing the vacuum electric field at the cathode edge relative to the vacuum electric field at the central portion of the cathode ${ }^{8}$ thereby reducing the local emitted current density. This addresses our practical goal of advanced cathode design as these mini-Pierce structures can greatly reduce nonuniformity in current density profile for SCL flows, minimizing damage and plasma formation while easing the task of matching into beam optics for advanced high voltage devices and applications.

\section{CONCLUSION}

Multidimensional space-charge-limited emission is important in many areas of plasma physics. Recently, much theoretical and computational effort has been spent on extending the well-known one-dimensional Child-Langmuir law to higher dimensions. From simple scaling laws through numerical solutions to the governing equations, multidimensional emission is becoming better characterized. Several interesting features emerge from this analysis. First, multidimensional effects can enhance both the global current and the local current density over traditional Child-Langmuir flows. The dominant physical quantity driving this enhancement can be shown to be the local vacuum electric field. Due to the importance of the vacuum electric field, Pierce-like cathode structures, even on the order of 1/100th of the gap spacing, can significantly repress these multidimensional effects. Furthermore, because of these enhancements, significant portions of the total current can be drawn with only a fraction of the cathode emitting. This results in a situation where the experimental area needed to infer the current density from the measured current for a given cathode is virtually unknown unless direct measurement of $J$ is possible. Finally, despite the advances detailed in this paper, the analytic solution to the multidimensional problem is still outstanding. This especially impacts our understanding of small $w / d$ emitting structures. Clearly, the asymptotic behavior as $w / d$ becomes small is of critical importance since real cathodes are dominated by extremely small cathode features (e.g., field-emitting tips).

\section{ACKNOWLEDGMENTS}

The authors would like to thank the following for their interest and important input in this work: Lt. J. Blahovec, Dr. K. L. Cartwright, Lt. C. S. Brodel, Dr. T. A. Spencer, Dr. R. E. Peterkin, Dr. M. D. Haworth, and Dr. D. S. Shiffler of AFRL; Dr. M. H. Frese of NumerEx; Professor R. M. Gilgenbach from The University of Michigan; Dr. A. Valfells of The University of Maryland; Dr. J. W. Schumer, Dr. M. Myers, and Dr. M. Friedman of the Naval Research Laboratory; Dr. S. Swanekamp of JAYCOR; and Dr. F. Hegeler of Commonwealth Technology, Inc.

This work was supported by the Air Force Office of Scientific Research (AFOSR). NumerEx' involvement is made possible through a contract with the Air Force Research Laboratory High Power Microwave Division, Contract No. F29601-94-C-0051, High Energy Plasma Theory and Experiments. Additional support by DUSD (S\&T) under the Innovative Microwave Vacuum Electronics MURI Pro- 
gram, managed by the AFOSR under Grant No. F49620-991-0297, and by the Northrop-Grumman Industrial Associates Program is gratefully acknowledged.

${ }^{1}$ C. D. Child, Phys. Rev. 32, 492 (1911).

${ }^{2}$ I. Langmuir, Phys. Rev. 2, 450 (1913).

${ }^{3}$ G. Jaffe, Phys. Rev. 65, 91 (1944).

${ }^{4}$ H. R. Jory and A. W. Trivelpiece, J. Appl. Phys. 40, 3294 (1969).

${ }^{5}$ Y. Y. Lau, D. Chernin, D. G. Colombant, and P. T. Ho, Phys. Rev. Lett. 66, 1446 (1991).

${ }^{6}$ J. W. Luginsland, Y. Y. Lau, and R. M. Gilgenbach, Phys. Rev. Lett. 77, 4668 (1996). There is a typo in this paper: the number 1 should be added to the right-hand side of Eq. (2).

${ }^{7}$ J. J. Watrous, J. W. Luginsland, and M. H. Frese, Phys. Plasmas 8, 4202 (2001).

${ }^{8}$ R. J. Umstattd and J. W. Luginsland, Phys. Rev. Lett. 87, 145002 (2001).

${ }^{9}$ Y. Y. Lau, Phys. Rev. Lett. 87, 278301 (2001).

${ }^{10}$ A. Valfells, D. W. Feldman, M. Virgo, P. G. O'Shea, and Y. Y. Lau, Phys. Plasmas 9, 2378 (2002).
${ }^{11}$ J. R. Pierce, Theory and Design of Electron Beams, 2nd ed. (Van Nostrand, New York, 1954), Chap. 10.

${ }^{12}$ J. M. Fine, T. M. Antonsen, Jr., and W. M. Manheimer, IEEE Trans. Plasma Sci. 16, 281 (1988).

${ }^{13}$ R. Barker and E. Schamloglu, Advances in HPM Technology (IEEEWiley, New York, 2001), Chaps. 9, 11.

${ }^{14}$ J. J. Watrous, J. W. Luginsland, and G. E. Sasser III, Phys. Plasmas 8, 289 (2001).

${ }^{15}$ M. D. Haworth et al., IEEE Trans. Plasma Sci. (submitted).

${ }^{16}$ For examples, see the Proceedings of the IEEE International Vacuum Microelectronics Conference, Davis, CA, 2001.

${ }^{17}$ J. W. Luginsland, S. McGee, and Y. Y. Lau, IEEE Trans. Plasma Sci. 26, 901 (1998).

${ }^{18}$ W. A. Anderson, J. Vac. Sci. Technol. B 11, 383 (1993).

${ }^{19}$ M. Myers and F. Hegeler, Naval Research Laboratory Cathode Conference (private communication).

${ }^{20}$ Tri-Comp suite of finite-element electromagnetic software available from Field Precision, P.O. Box 13595, Albuquerque, NM 87192 (also see http:// www.fieldp.com/). 